\title{
Robust PID Stabilization of Linear Neutral Time-Delay Systems
}

\author{
H. Farokhi Moghaddam, N. Vasegh
}

\author{
Hassan Farokhi Moghadam* \\ Faculty of Electrical and Computer Engineering \\ Shahid Rajaee Teacher Training University \\ Lavizan, Tehran, Iran. \\ *Corresponding author: hassan.farokhi89@gmail.com
}

\author{
Nastaran Vasegh \\ Faculty of Electrical and Computer Engineering \\ Shahid Rajaee Teacher Training University \\ Lavizan, Tehran, Iran. \\ E-mail: n.vasegh@srttu.edu
}

\begin{abstract}
This paper deals with the problem of robust stabilization of neutral timedelay systems using Proportional-Integral-Derivative (PID) controller. A graphical approach which will obtain the set of robust stabilizing PID controllers is presented. The main point in this approach is the frequency response of the neutral system, which can effectively reduce the mathematical complexities of system modeling. It is shown that our the proposed method is effective and useful for many of real control systems deal with time-delays and parametric uncertainties. It is illustrated by an example at the end.
\end{abstract}

Keywords: Robust stabilization, PID controller, Time-delay, Neutral systems.

\section{Introduction}

Proportional-Integral-Derivative (PID) controllers have become the best known controllers since their entrance to industrial applications and are used frequently due to their simplicity and good performance [1]. Despite their wide application, determining of all stabilizing regions of PID controllers has been studied just only in the last decade [2]. In [3, 4], a mathematical generalization of the Hermite-Biehler theorem to find all stabilizing PID controllers for systems with time-delay has been used. In [5], the entire region of PID controller has been obtained for linear time-delay systems. In recent years, the robust stabilization of neutral time-delay systems has received considerable attention and has been has been the focus of much research $($ see $[6,7]$ and references within).

In this paper, a graphical PID controller design approach for control systems with neutral time-delay is proposed which guarantees the required stabilizing margins of the closed loop system for the uncertain system. Basic knowledge of robust control theory seems sufficient in this proposed approach and the method is easy to understand. Therefore, simplicity is the main characteristic of the approach. The results of the simulations show the effectiveness of proposed method.

The rest of the paper is organized as follows. Section 2 states the problem and preliminary. In section 3 the nominal and robust stability criterion are discussed. All different stabilizing regions which will ensure nominal and robust stability are determined in section 4. Simulation results which will show the correctness of the design approach are shown for an illustrative example in section 5. Finally, results of this paper are summarized in section 6 . 


\section{Problem Statement}

The most general form of PID controller is the second order system in the s-domain defined by

$$
K(s)=k_{p}+\frac{k_{i}}{s}+k_{d} s .
$$

In this paper, this controller is implemented for a linear neutral delayed system with the following general transfer function form:

$$
G(s)=\frac{r(s)}{p(s)+q(s) e^{-T s}}
$$

where $T$ is time-delay $p(s), q(s)$ and $r(s)$ are real polynomials. According to [8], the system is of neutral type if degrees of $p(s)$ and $q(s)$ are the same.

PID Robustness of this type of system is the main consideration in this paper. Although there are different forms of model uncertainty [9], but an additive uncertainty modeling structure which is shown in Figure 1, is chosen in this paper as this uncertainty description is closely related to the transfer function representation of system.

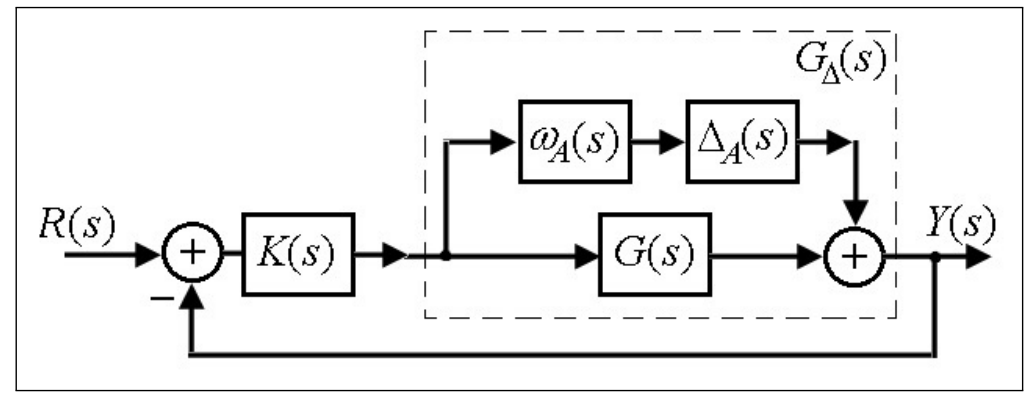

Figure 1: Feedback system with additive uncertainty [9]

Here $K(s)$ represents the PID controller defined by transfer function in (1), the input signal is $R(s)$ and the output signal is $Y(s), G_{\triangle}(s)$ is the uncertain system, $G(s)$ is the nominal system without uncertainty, $\omega_{A}(s)$ means the additive weight and is a rational transfer function in this study and $\Delta_{A}(s)$ is a stable transfer function so that $\forall s\left|\Delta_{A}(s)\right| \leq 1$.This kind of uncertainty can be represented by following equation in frequency domain approach.

$$
G_{\triangle}(j \omega)=G(j \omega)+\omega_{A}(j \omega) \Delta_{A}(j \omega), \quad\left|\Delta_{A}(j \omega)\right| \leq 1 \forall \omega .
$$

\section{Stabilization of Linear Uncertain Neutral Delayed systems}

In this section, we study the mathematical formulations for the nominal and robust stability problem in details in following sub-sections.

\subsection{Nominal Stability of Linear Neutral Delayed Systems}

In the nominal case, based on standard unity feedback like Figure 1 (without uncertainty), the characteristic equation in frequency domain $(s=j \omega)$, can be written as

$$
\Delta(j \omega)=1+K(j \omega) G(j \omega)
$$


PID controller and transfer function form at (1-2) can be represented as

$$
\begin{aligned}
K(j \omega) & =k_{p}+j\left(k_{d} \omega-\frac{k_{i}}{\omega}\right), \\
G(j \omega) & =\frac{N((j \omega))}{D(j \omega)}=\frac{r(j \omega)}{p(j \omega)+q(j \omega)(\cos (T \omega)+j \sin (T \omega))} .
\end{aligned}
$$

Let $M(j \omega)=N(j \omega) D(-j \omega)$, so

$$
G(j \omega)=\frac{\operatorname{Re}(M(j \omega))+j \operatorname{Im} M(j \omega)}{|D(j \omega)|^{2}}=\frac{R_{M}(j \omega)+j I_{M}(j \omega)}{|D(j \omega)|^{2}} .
$$

Therefore (4) can be written as

$$
\Delta(j \omega)=1+\left(k_{p}+j\left(k_{d} \omega-\frac{k_{i}}{\omega}\right)\right)\left(\frac{R_{M}(j \omega)+j I_{M}(j \omega)}{|D(j \omega)|^{2}}\right) .
$$

The numerator becomes

$$
\begin{array}{r}
\omega k_{p} R_{M}(j \omega)+k_{i} I_{M}(j \omega)-k_{d} \omega^{2} I_{M}(j \omega)+\omega|D(j \omega)|^{2}+ \\
j\left(k_{p} \omega I_{M}(j \omega)-k_{i} R_{M}(j \omega)+k_{d} \omega^{2} R_{M}(j \omega)\right)
\end{array}
$$

Now all stabilizing regions can be determined like what is shown in Figure 2 (Right). Note that we complete the calculations here just for a constant value of $k_{d}$ in the $\left(k_{p}, k_{i}\right)$ plane, for brevity. The $\left(k_{p}, k_{d}\right)$ plane for a constant value of $k_{i}$ is obtained similarly, but for the $\left(k_{i}, k_{d}\right)$ plane at a constant value of $k_{p}$, nominal and robust stability cannot be obtained directly by (15) and (21) in the next section. For more accuracy, based on [10], for constant value of $k_{i}$ and $k_{d}$ stability boundaries are defined in the $\left(k_{p}, k_{i}\right)$ plane and $\left(k_{p}, k_{d}\right)$ plane, respectively and then the lines that define the boundary of stability are extracted. The illustrative example in section 5 shows the effectiveness of the approach.

\subsection{Robust Stability of Linear Neutral Delayed Systems}

In order to analyze the stability in presence of uncertainty nominal system should be stable. For getting robust stability in this condition, following theorem is stated from [9].

Theorem 1. Small gain theorem: If $L(s)$ is a system with a stable loop transfer function, then the closed-loop system is stable if

$$
\|L(j \omega)\|_{\infty}<1, \quad \forall \omega
$$

where $L(s)=K(s) G(s)$.

As a result from this theorem, if $\|L(j \omega)\|_{\infty}<1$ the closed loop system is stable. Using this theorem and uncertainty structure in Figure 1, it is usually said that if system is robustly stable, the following constraint is met for additive uncertainty:

$$
\left\|\frac{\omega_{A}(j \omega) K(j \omega)}{1+K(j \omega) G(j \omega)}\right\|_{\infty} \leq \gamma, \quad \forall \omega
$$

For robust case, we can rewrite (6) as:

$$
\Delta_{R}(j \omega)=1+K(j \omega) G(j \omega)-\frac{1}{\lambda}\left(\omega_{A}(j \omega) K(j \omega)\right) .
$$

The rational function can be written as:

$$
\omega_{A}(j \omega)=R_{\omega}(\omega)+j I_{\omega}(\omega) .
$$

Then by continuing calculation in (9), the numerator of (12) can be simplified as

$$
\Delta_{R}(j \omega)=\operatorname{Re}\left(\Delta_{R}(j \omega)\right)+j \operatorname{Im}\left(\Delta_{R}(j \omega)\right) .
$$




\section{All Stabilizing PID Controller Regions}

The following steps are proposed to find the boundaries of all stabilizing PID controller.

1. Decompose the frequency form of the system without delay and uncertainty into real and imaginary parts, and substitute them into (5)-(9) to obtain the parameters.

2. Analyze the neutral system in presence of time-delay and additive uncertainty, and do the previous step again. (Eqs. (5)-(14) should be considered)

3. Determine the PID nominal and robust stability boundaries of the system using their related equations in this section, respectively.

4. Finally, plot all the different stabilizing regions.

Here based on the step 3, we'll complete equations obtained in the previous section. For the nominal case, by putting (10) equal to zero, we have

$$
\begin{aligned}
& k_{p} \omega R_{M}(j \omega)+k_{i} I_{M}(j \omega)-k_{d} \omega^{2} I_{M}(j \omega)+\omega|D(j \omega)|^{2}=0, \\
& k_{p} \omega I_{M}(j \omega)-k_{i} R_{M}(j \omega)+k_{d} \omega^{2} R_{M}(j \omega)=0 .
\end{aligned}
$$

which results in

$$
\left.k_{p}(\omega)\right)=-\frac{|D(j \omega)|^{2} R_{M}(j \omega)}{|M(j \omega)|^{2}},
$$

and

$$
\left.k_{i}(\omega)\right)=k_{d} \omega^{2}-\frac{\omega|D(j \omega)|^{2} I_{M}(j \omega)}{|M(j \omega)|^{2}} .
$$

Since

$$
R_{M}(j \omega)=|M(j \omega)| \cos (\theta(\omega)) \quad I_{M}(j \omega)=|M(j \omega)| \sin (\theta(\omega))
$$

where $\theta(\omega)=\angle G(j \omega)$, (16) and (17) can be simplified as

$$
\left.k_{p}(\omega)\right)=-\frac{|D(j \omega)|^{2} \cos (\theta(\omega))}{|M(j \omega)|},
$$

and

$$
\left.k_{i}(\omega)\right)=k_{d} \omega^{2}-\frac{\omega|D(j \omega)|^{2} \sin (\theta(\omega))}{|M(j \omega)|} .
$$

Robust consideration yields

$$
\begin{aligned}
& \left(\omega R_{M}(j \omega)-\frac{\omega|D(\omega)|^{2}}{\lambda} R_{\omega}(\omega)\right) k_{p}+\left(I_{M}(j \omega)-\frac{|D(j \omega)|^{2}}{\lambda} I_{\omega}(\omega)\right) k_{i}= \\
& \left(I_{M}(j \omega)-\frac{|D(\omega)|^{2}}{\lambda} I_{\omega}(\omega)\right) \omega^{2} k_{d}-\omega|D(j \omega)|^{2}, \\
& \left(\omega I_{M}(j \omega)-\omega \frac{|D(\omega)|^{2}}{\lambda} I_{\omega}(\omega)\right) k_{p}+\left(-R_{M}(j \omega)+\mid \frac{\left.D(j \omega)\right|^{2}}{\lambda} R_{\omega}(\omega)\right) k_{i}= \\
& \left(\frac{\left.D(j \omega)\right|^{2}}{\lambda} R_{\omega}(\omega)-R_{M}(j \omega)\right) \omega^{2} k_{d} .
\end{aligned}
$$

which implies

$$
\left.k_{p}(\omega)\right)=-\frac{|D(j \omega)|^{2}\left(R_{M}(j \omega)-\frac{\left.D(j \omega)\right|^{2}}{\lambda} R_{\omega}(\omega)\right)}{Q(j \omega)},
$$


and

$$
\left.k_{i}(\omega)\right)=\omega^{2} k_{d}-\frac{\omega|D(j \omega)|^{2}\left(I_{M}(j \omega)-\frac{\left.D(j \omega)\right|^{2}}{\lambda} I_{\omega}(\omega)\right)}{Q(j \omega)},
$$

where

$$
Q(j \omega)=|M(j \omega)|^{2}+\left|\omega_{A}(j \omega)\right|^{2}-2 \frac{\left.D(j \omega)\right|^{2}}{\lambda}\left(R_{M}(j \omega) R_{\omega}(\omega)+I_{M}(j \omega) I_{\omega}(\omega)\right) .
$$

which can be simplified using (18) if necessary. Using (21)-(24), the nominal stability boundary and robust stability region are obtained in the $\left(k_{p}, k_{i}\right)$ plane. The approach is explained in the following illustrative example.

\section{Illustrative Example}

In this example we consider the neutral time-delay system with following transfer function considered in $[8]$.

$$
G(s)=\frac{1}{(s+1)^{4}\left(s+1+s e^{-s}\right)}
$$

The range of the unknown time-delay is considered as $T \in[0.5,1.5]$. So the uncertain model of the system is $G_{\Delta}(s)=\frac{1}{(s+1)^{4}\left(s+1+s e^{-T s}\right)}$. For this system, we can write (3) as,

$$
\left|\frac{G_{\Delta}(s)-G(s)}{\omega_{A}(s)}\right| \leq 1, \quad \forall \omega .
$$

Therefore, it is required to find

$$
\left|\omega_{A}(j \omega)\right| \geq\left|G_{\Delta}(j \omega)-G(j \omega)\right|
$$

where

$$
\left|G_{\Delta}(j \omega)-G(j \omega)\right|=\left|\frac{1}{j \omega+1)^{4}}\right|\left|\frac{1}{j \omega+1+j \omega e^{-j \omega T}}-\frac{1}{j \omega+1+j \omega e^{-j \omega \bar{T}}}\right| .
$$

For determining the $\omega_{A}(s)$, at first we plot the additive uncertainty which is the right part of inequality and is shown in Figure 2 and then based on approximate estimation, transfer function form is determined. MATLAB/SISOTOOL is used to facilitate this choice. The MATLAB/cftool can also be used by fitting a good rational function. Here it is obtained as

$$
\omega_{A}(s)=\frac{1.6 s^{2}}{(s+1)^{4}(s+2)} .
$$

This is specified in Figure 2 (Left) which clearly covers the entire uncertainty region. Then the all PID stabilizing regions for this system with $T=0.5$ are shown in Figure 2 (Right) which is obtained as a result of analysis done in previous sections. Figure 4 (Right side) shows the $\left(k_{i}, k_{d}\right)$ plane for $k_{p}=0.2$. The intersection of all regions inside the nominal stability boundary of the $\left(k_{p}, k_{i}\right)$ plane is the robust stability region.

To check the correctness of stabilizing regions in Figures 6 and 7, two points from each plane are chosen: one is selected from the robust stability region and another one is selected from nominal region (outside this region). Then the transfer functions of PID controllers are extracted and the constraint criterion in (11) is checked by Bode plot. For example in the $\left(k_{p}, k_{i}\right)$ plane in Figure 5 (Left), the robust chosen point is $(1.02,0.301)$ and another one is $(2.47,0.298)$. Their related PID controllers are:

$$
K_{1}(s)=1.02+\frac{0.301}{s}+0.3 s, \quad K_{2}(s)=2.47+\frac{0.298}{s}+0.3 s
$$




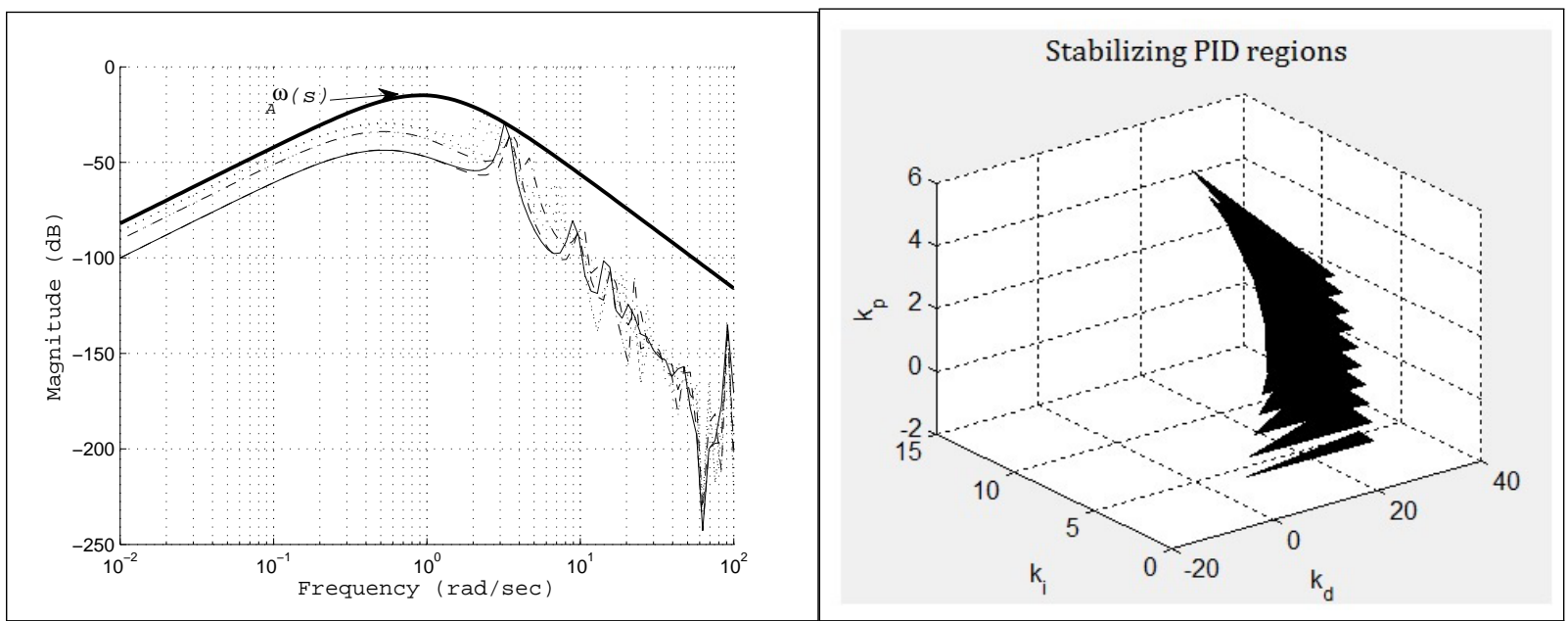

Figure 2: Left; Uncertainty region and the additive uncertainty weight. Right; All nominal stabilizing PID regions in the case $T=0.5$.

Now it can be seen that for robust $K_{1}(s)$, we have $\left\|\frac{\omega_{A}(j \omega) K_{1}(j \omega)}{1+K_{1}(j \omega) G(j \omega)}\right\|_{\infty}=0.2476$ but for $K_{2}$, it results in $\left\|\frac{\omega_{A}(j \omega) K_{2}(j \omega)}{1+K_{2}(j \omega) G(j \omega)}\right\|_{\infty}=1.4>1$. It is clear that our expectation has been satisfied. In Figure 5 (left side), their magnitude is shown for different frequencies which is verified these calculations. Repeating this procedure in MATLAB shows that any controller selected from inside the robust stability region is capable of robust stabilization of the system.

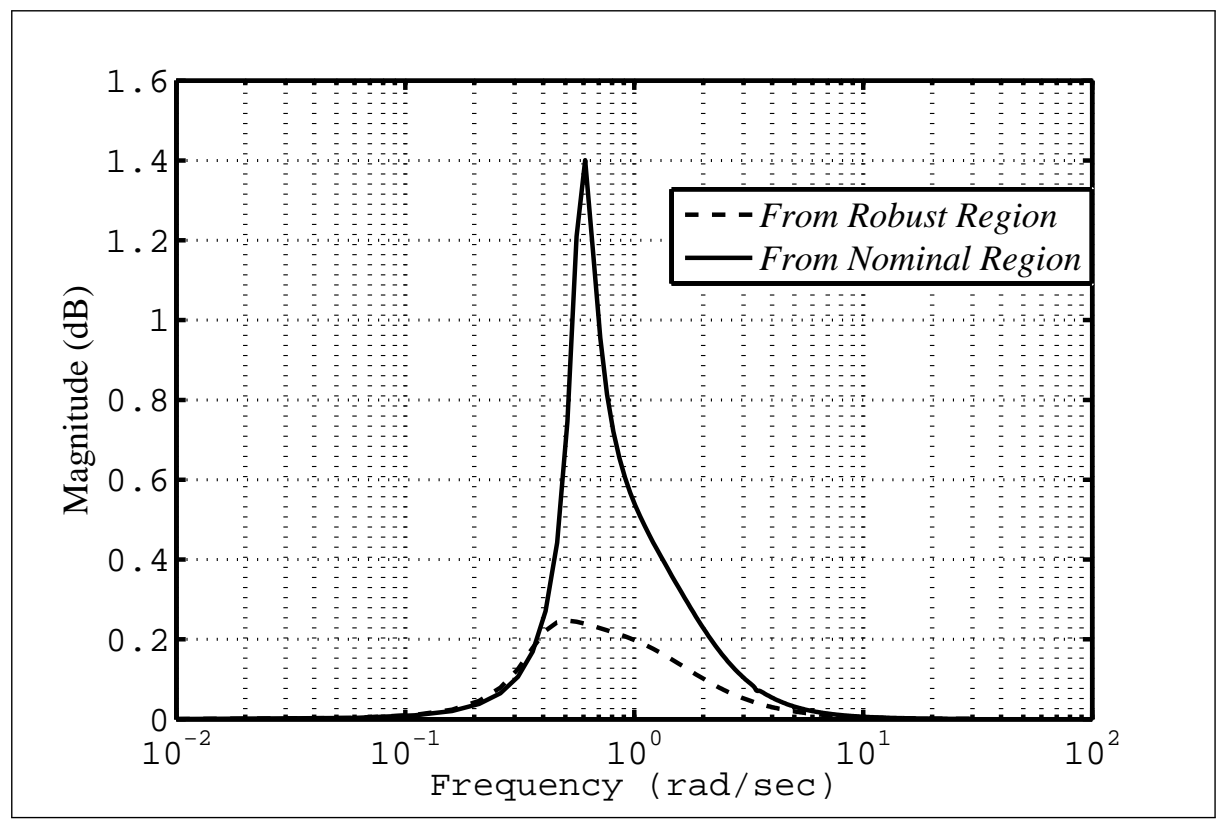

Figure 3: Magnitude of two different nominal and robust points.

Step response of the closed loop system for the entire unknown time-delay range is also plotted in the left side of Figure 4 to show the stability and good performance of robust system. The nominal and the robust stability region in the $\left(k_{p}, k_{d}\right)$ plane for $k_{i}=0.5$ and in the $\left(k_{p}, k_{i}\right)$ plane for $k_{d}=0.3$ are also shown in Figure 5 (right and left), respectively. 

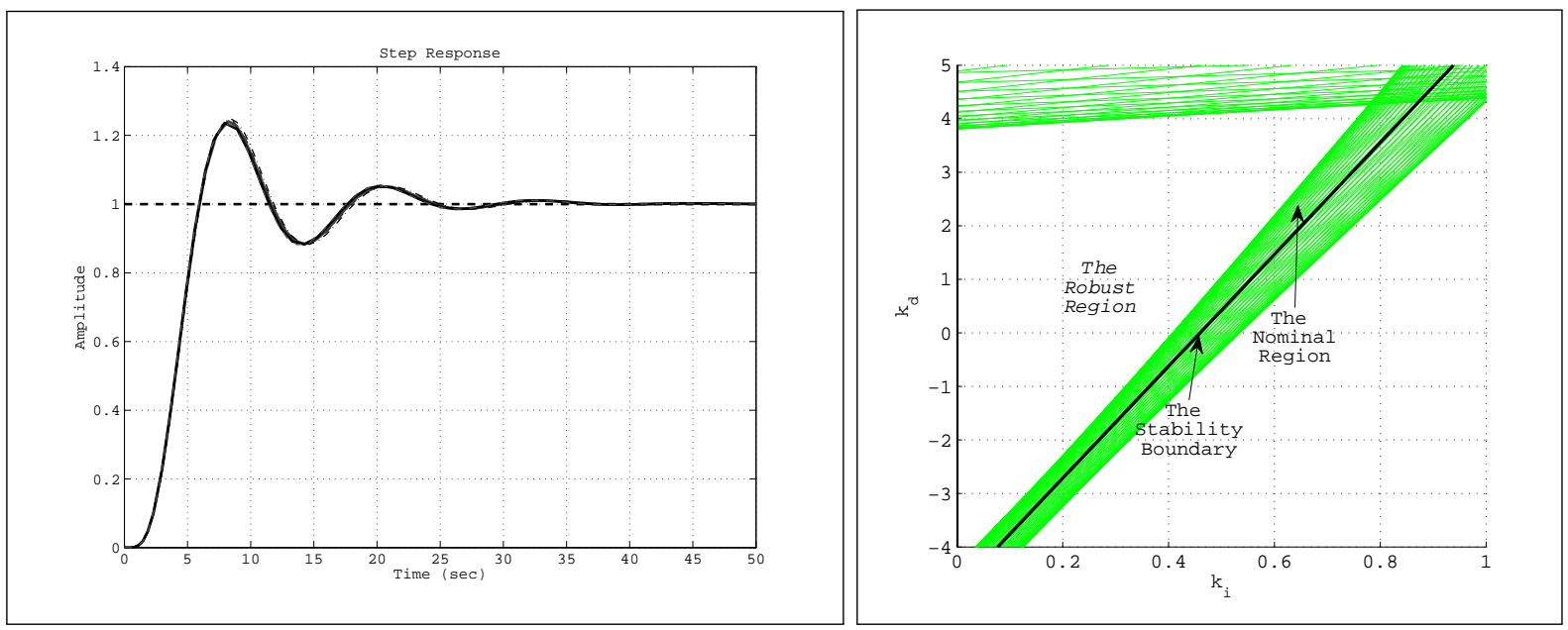

Figure 4: Left; Closed loop step response for entire uncertainty range. Right; The stabilizing region in the $\left(k_{i}, k_{d}\right)$ plane for $k_{p}=0.2$.
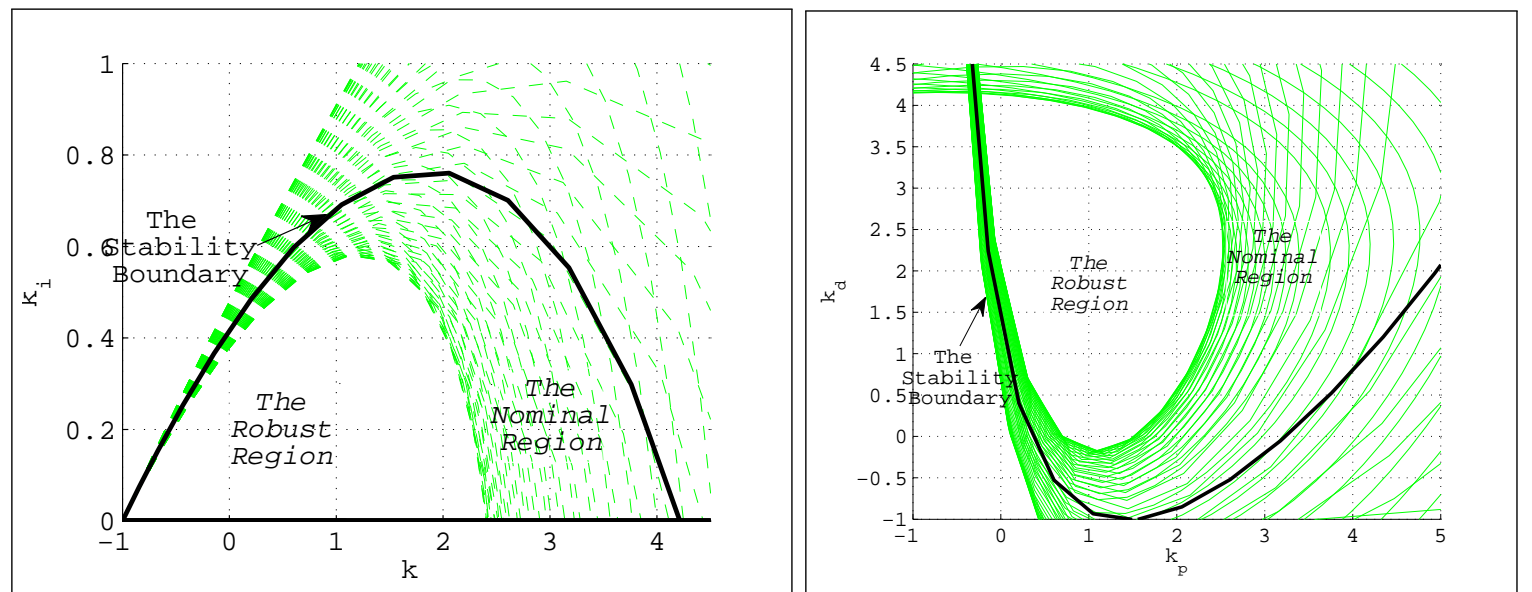

Figure 5: The nominal and robust stability region: Left; $\left(k_{p}, k_{i}\right)$ plane for $k_{d}=0.3$. Right; $\left(k_{p}, k_{d}\right)$ plane for $k_{i}=0.5$.

\section{Conclusion}

In this paper, a graphical design approach was introduced for finding all PID stabilizing regions of uncertain neutral time-delay system. This proposed design method was based on the frequency response of the system and it can reduce the complexities involved in system modeling. An example for illustrating this approach was studied. The results were satisfactory and the nominal and robust stability regions were determined.

\section{Bibliography}

[1] O.T. Altinoz, A.E. Yilmaz, G.-W. Weber (2012), Application of Chaos Embedded PSO for PID Parameter Tuning, Int J Comput Commun, ISSN 1841-9836, 7(2):204-217.

[2] B. Fang (2010), Computation of stabilizing PID gain regions based on the inverse Nyquist plot, J Proc Cont, 20:1183-1187. 
[3] K. W. Ho, A. Datta, S.P. Bhattacharya (1999), Generalizations of the Hermite-Biehler theorem, Lin Alg its App, Vol. 302-303, pp. 135-153.

[4] K. W. Ho, A. Datta, S.P. Bhattacharya (2003), PID stabilization of LTI plants with timedelay, Proceeding 42nd IEEE Conference on Decision and Control, 4038-4043.

[5] N. Hohenbichler (2009), All stabilizing PID controllers for time-delay systems, Automatica, 45:2678-2684.

[6] L. Dugard, E. I. Verriest (1998), Stability and Control of Time-delay Systems, London: Springer-Verlag.

[7] O.M. Kwon, J.H. Park, S.M. Lee (2008), On stability criteria for uncertain delay-differential systems of neutral type with time-varying delays, AppL Math Compu, 197:864-873.

[8] J. R. Partingtona, C. Bonnet (2004), $\mathrm{H} \infty$ and BIBO stabilization of delay systems of neutral type, Syst 8 Cont Lett, 52:283-288.

[9] S. Skogestad, I. Postlethwaite (2001), Multivariable Feedback Control, John Wiley \& Sons.

[10] G.J. Silva, A. Datta, S.P. Battacharrya (2005), PID Controllers for Time-Delay Systems, Birkhauser, Boston, 2005. 